\title{
The gut microbiota and mucosal T cells
}

\author{
Patrick M. Smith ${ }^{1,2}$ and Wendy S. Garrett ${ }^{1,2,3,4,5 *}$ \\ 1 Department of Immunology and Infectious Diseases, Harvard School of Public Health, Boston, MA, USA \\ 2 Department of Genetics and Complex Diseases, Harvard School of Public Health, Boston, MA, USA \\ ${ }^{3}$ Department of Medicine, Harvard Medical School, Boston, MA, USA \\ ${ }^{4}$ Department of Medical Oncology, Dana-Farber Cancer Institute, Boston, MA, USA \\ 5 The Broad Institute of MIT and Harvard, Cambridge, MA, USA
}

\section{Edited by:}

Peter J. Turnbaugh, Harvard University, USA

\section{Reviewed by:}

Dennis L. Kasper, Harvard Medical

School, USA

Daniel Peterson, University of

Nebraska-Lincoln, USA

\section{*Correspondence:}

Wendy S. Garrett, Department of Immunology and Infectious Diseases, Harvard School of Public Health, 665 Huntington Avenue, SPH 1, 9th Floor, Boston, MA 02115, USA.

e-mail:wgarrett@hsph.harvard.edu
It is intuitive that immune cells in the gut may require microbiota-derived cues for their differentiation. The proximity between host and microbe in the intestine would seemingly necessitate co-adaptation. However, it has been challenging to determine the members and features of the gut microbiota that influence immune system development and function. The recent identification of immunomodulatory members of the commensal microbiota is providing insight into the dependence of select, intestinal immune cell subsets on specific microbial species. In this review, we focus on the gut microbiota's influence on the development and function of mucosalT cells subsets, specifically intraepithelial lymphocytes and lamina propria CD4T cells.

Keywords: gut microbiota, T cells, mucosal immunity

\section{OVERVIEW}

The mucosal immune system of the intestinal tract faces the challenge of co-existing with a diverse and dynamic community of microbes while remaining poised to protect and defend against invasive pathogens. This microbiota is an active participant in host metabolism and also provides critical developmental cues for the immune system. Studies from animals models lacking all microbes, referred to as germ-free, have established that gut microbes are crucial for the development, maturation, and function of the intestinal immune system and many aspects of systemic immunity. Mucosal $\mathrm{T}$ cells that reside within the epithelial cell layer and the lamina propria (LP) of the intestine are especially reliant on direct and indirect microbial signals for their proper differentiation and function. Recently a few species, Bacteroides fragilis, Candidatus Arthromitus sp. segmented filamentous bacteria (SFB), and Clostridium spp. from Clostridium Clusters III, IV, and XIVa have been identified that drive both effector and/or regulatory mucosal $\mathrm{T}$ cell maturation. In this review, we provide an introduction to mucosal T cell subsets, specifically intraepithelial lymphocytes and LP CD4 T cells, with a focus on the gut microbiota's influence on their development and function.

\section{INTRAEPITHELIAL T LYMPHOCYTES}

Below the tight junctions that join the plasma membranes of epithelial cells and residing above the basement membrane, are specialized subsets of gut $\mathrm{T}$ cells aptly named intraepithelial cell lymphocytes (IELs; Figure 1). IELs promote barrier repair, rapidly accumulate at sites of injury and infection, and defend against intestinal pathogens, such as Eimeria vermiformis (Roberts et al., 1996) and Salmonella spp. (Dalton et al., 2006). The majority of IELs express the CD8 glycoprotein, that binds MHC class I molecules (van Wijk and Cheroutre, 2009). IELs are categorized by the two chains that compose their $\mathrm{T}$ cell receptor: either alpha beta $(\alpha \beta)$ or gamma delta $(\gamma \delta)$ heterodimers. $\alpha \beta$ IELs are primarily derived in the thymus and migrate into the intestine after development (Sheridan and Lefrancois, 2010). In contrast, $\gamma \delta$ T cells are derived extrathymically and while they constitute a small fraction of $\mathrm{T}$ cells in peripheral lymph nodes and the spleen, $50 \%$ of IELs in mice express $\gamma \delta$. In humans, $15 \%$ of IELs in the small intestine (SI) and upward of $40 \%$ in the colon are $\gamma \delta$ positive (Kagnoff, 1998). In response to bacteria or intestinal injury, $\gamma \delta$ IELs produce proinflammatory cytokines and chemokines that recruit neutrophils, eosinophils, and T cells. $\gamma \delta$ IELs also promote epithelial healing via production of keratinocyte growth factor, which stimulates epithelial cell proliferation and restoration of barrier function (Yang et al., 2004).

Despite the important role of $\gamma \delta$ IELs in the intestine and their close proximity to luminal and mucosal microbes, relatively little was known about their regulation by the gut microbiota until recently. Because of their small absolute numbers and propensity for apoptosis when cultured ex vivo, $\gamma \delta$ IELs have proven a challenging cell subset to study. The application of laser capture microdissection to the study of $\gamma \delta$ IELs by the Hooper laboratory has provided a major advance for investigating this cell subset (Ismail et al., 2009). While germ-free (GF) mice have equal numbers of $\gamma \delta$ IELs compared with conventionally raised mice (Bandeira et al., 1990), data have emerged on the significant role of the gut microbiota on $\gamma \delta$ IEL function. Ismail et al. (2009) have begun to unravel an elaborate and dynamic cross-talk between commensal bacteria and $\gamma \delta$ IELs during mucosal injury that both promotes wound healing and prevents invasion by opportunistic pathogens. By comparing the transcriptional profiles of colonic $\gamma \delta$ IELs isolated from conventional and GF mice both prior to and following injury with the mucosal disruptant, dextran sulfate sodium (DSS), Ismail et al. (2009) identified key effectors in the $\gamma \delta$ IEL microbiota-dependent response to injury. While upregulation of lysozyme was a microbiota-independent function of IELs, the 
induction of numerous chemokines (including KC and MIP2 $\alpha$ ), proinflammatory cytokines (e.g., IL-1 $\beta$ ), and the bactericidal lectin RegIII $\gamma$ was microbiota-dependent. Importantly, colonization of GF mice with a specific pathogen-free (SPF) microbiota restored $\gamma \delta$ IELs' ability to promote wound healing. Furthermore, $\gamma \delta$ IELs were also critical to protect against bacterial invasion immediately following intestinal injury as increased numbers of bacteria were detected in mesenteric lymph nodes in mice deficient in IELs (Ismail et al., 2009).

$\gamma \delta$ IELs exhibit a multifaceted ability to promote intestinal homeostasis by responding directly to signals from the microbiota. The response of $\gamma \delta$ IELs to gut microbes is only partially dependent on TLR signaling. Thus, it will be interesting to determine the other receptors, microbiota associated molecular patterns and specific bacterial species involved in orchestrating the IEL immune response to mucosal injury. Given that $\gamma \delta$ IELs produce cytokines, such as IL- $1 \beta$ and TGF $\beta$, that induce expansion of both antimicrobial and anti-inflammatory LP CD4 T cell subsets, respectively, it will also be interesting to determine how cross-talk between these two populations regulates intestinal homeostasis following injury and subsequent bacterial invasion.

\section{LAMINA PROPRIA CD4 T LYMPHOCYTES}

Beneath the epithelial cell layer's basement membrane is the LP region of the mucosa. The LP of the small and large intestine is home to $70-80 \%$ of the body's immune cells (Furness et al., 1999). As opposed to the intraepithelial lymphoid compartment, populated with CD8 T cells, the LP is dominated by T cells expressing the CD4 glycoprotein, which binds MHC class II. While there are CD8 $\alpha \beta$ and $\gamma \delta$ LP populations, we focus on the many helper and regulatory CD4 T cells that reside in the LP and the evolving understanding of their relationship with gut microbiota.

Helper CD4 T cells (Th) represent a diverse collection of subsets with specific cytokines and chemokine profiles that result in activation and growth of cytotoxic T cells, promotion of myeloid cell bacte-

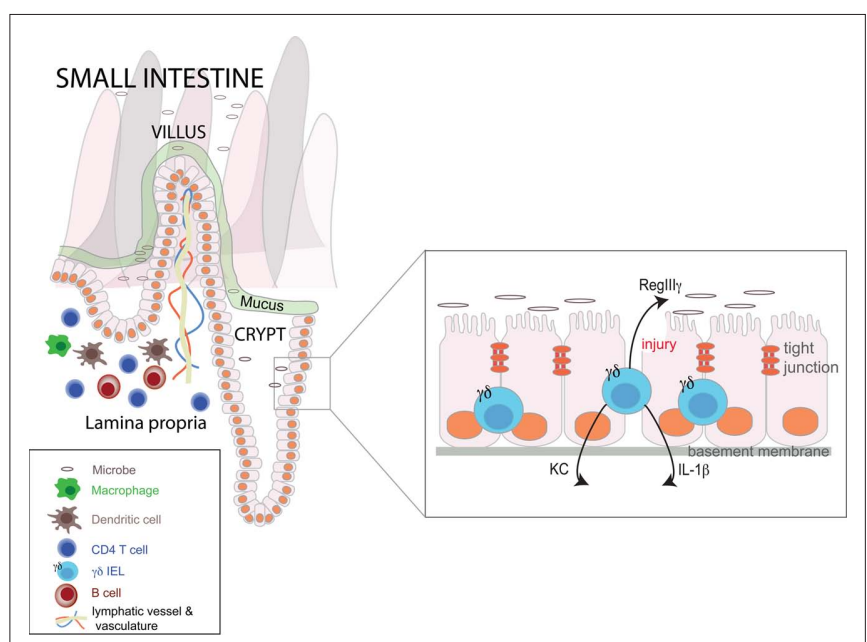

FIGURE 1 |A schema of the small intestinal mucosa. The crypt-villus axis of the small intestine is illustrated, as are its key immune subsets. Inset is an expanded view of the epithelium highlighting the $\gamma \delta$ intraepithelial lymphocytes and several of the microbiota-dependent factors they produce in response to injury. ricidal activity, and $\mathrm{B}$ cell differentiation and antibody production. In general, Thelper type 1 produce interferon- $\gamma$, TNF- $\alpha$, and IL- 12 and participate in host defense against intracellular pathogens; $T$ helper type 2 produce IL-10, IL-13, IL-5, and IL-4 and defend against helminths; and T helper 17 produce IL-17, IL-21, and IL-22 and afford protection against extracellular bacteria and parasites. Interactions between LP CD4 T cells and the gut microbiota, often with epithelial cells or dendritic cells functioning as the intermediaries, are critical for shaping the adaptive immune response in the intestine (Figure 2).

In healthy individuals all of these Th subsets are present in the LP and exist in a dynamic balance with regulatory CD4 T cells. Regulatory T cells also have many subsets especially in the gut. The environmental exposures (dietary components, resident intestinal microbes, transiently ingested microbes, and invasive pathogens) that the gut continuously encounters necessitate a nimble, diverse, and versatile functional potential of the adaptive T lymphoid compartment. There is also an increasing understanding of the functional plasticity across CD4 subsets, especially for regulatory subsets and Th17 cells (Lee et al., 2009).

Both the gut and systemic immune system receive critical developmental cues from the intestinal microbiota. For example, GF rodents have marked abnormalities in mesenteric lymph nodes, peyer's patches, and splenic architecture (Smith et al., 2007). In addition to impacting lymphoid structures and cell numbers, the microbiota influence lymphoid function under steady state conditions and in response to injury without any direct physical interaction. Surveys of the gut bacterial communities are revealing that there are alterations in the microbiota in both system and gutlocalized allergic and inflammatory disease (Cerf-Bensussan and Gaboriau-Routhiau, 2010). As T cells play critical effector roles in diseases like asthma, inflammatory bowel disease, type 1 diabetes, and multiple sclerosis, it is of great import to unravel the cause and effect relationships between the gut microbiota and LP T cell responses in allergic and autoimmune diseases.

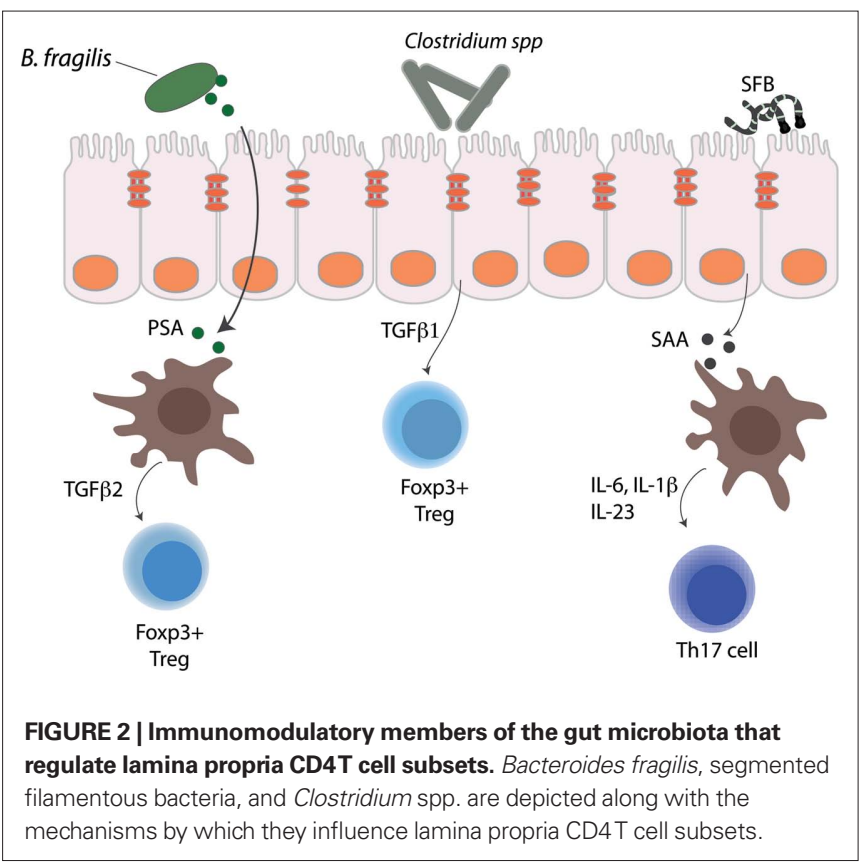




\section{GUT MICROBIOTA AND Th1-Th2 CELL EQUILIBRIUM}

T helper type 1 (Th1) and type 2 (Th2) balance has been a useful paradigm facilitating understanding of both how the immune system executes cellular and humoral immune responses and how imbalanced and over-exuberant responses underlie arthritis, type 1 diabetes and multiple sclerosis in the case of Th1 immunity or asthma and allergic diseases in the case of Th2 immunity (Mosmann et al., 1986; Zhu et al., 2010). With the discovery of other T helper subsets, e.g., Thelper 17 and T helper 9 and also regulatory subsets, the binary model of Th1 vs. Th2 is overly simplistic. However, early focus on these subsets during development and in GF mice was instrumental in understanding how the microbiota influence immune cell development. During the neonatal period; the immature fetal immune system is biased toward a Th2 phenotype, which if not corrected predisposes rodents and humans to infections, allergic and inflammatory diseases (Wilson, 1986; Holt, 1995). Prior to weaning, rats are skewed toward a Th2 biased phenotype in the spleen and mesenteric lymph node and lack basic Th1 cell mediated functions. These defects can be corrected by a lyophilized combination of bacteria including Staphylococcus, Streptococcus, and Klebsiella strains (Bowman and Holt, 2001). Recent studies in lymphopenic mice have provided mechanistic insight demonstrating that TLR ligands derived from the intestinal microbiota drive spontaneous $\mathrm{T}$ cell proliferation via innate cell production of IL-6 (Feng et al., 2010). These data suggest that exposure to bacteria may be necessary to drive physiologic Th1 cell development. Subsequent studies have revealed that GF mice have reduced numbers of Th1 cells in both systemic and mucosal compartments (Smith et al., 2007). This reduction in basal Th1 immunity contributes to susceptibility to infection and morbidity with bacterial pathogens, including Shigella flexneri and Listeria monocytogenes (Round and Mazmanian, 2009). These early studies focused on Th1 and Th2 cells established an important role for the gut microbiota in CD4 T cell development systemically and within the gut.

While the human gut is home to trillions of bacteria cells representing a thousand species, $B$. fragilis was the first bacterial species identified that corrected the Th1 and Th2 cell imbalances observed in non-gut lymphoid tissues in GF mice. $B$. fragilis is a Gram-negative symbiont that inhabits the lower intestinal tract and colonizes an estimated 30-70\% of humans (Sears and Pardoll, 2011). Monocolonization of GF mice with $B$. fragilis induced overall CD4 T cell expansion and increased numbers of Th1 cells to levels similar to those of conventionally raised mice. Mechanistically, the effect of $B$. fragilis was entirely dependent on a zwitterionic capsular polysaccharide, polysaccharide A (PSA; Mazmanian et al., 2005). Exposure to PSA in vivo and in vitro was sufficient to induce Th1 expansion and cytokine production and GF mice colonized with B. fragilis lacking PSA failed to correct their Th1/Th2 imbalance. As all humans and mice do not necessarily have $B$. fragilis in their gut, the intestinal microbiota undoubtedly harbors other symbiotic factors that facilitate systemic and intestinal Th1 cell development yet to be discovered.

\section{GUT MICROBIOTA AND Th17 CELLS}

Early studies aimed at understanding how the gut microbiota affect LP CD4 T cell populations were mainly focused on the restoration of Th1 responses; more recent work has shed light on how commensal bacteria also affect the development of Th17 cells. Th17 cells were identified as major mediators of pathology in several autoimmune and inflammatory disease models; however, Th17 cells play an important role in host defense against extracellular bacteria and parasites (Korn et al., 2009). Th17 cells are found both in the LP of the SI and the colon and have an established role from studies in mouse models in defense against Shigella flexneri and Citrobacter rodentium (Ivanov et al., 2009; Sellge et al., 2010). Similar to findings with LP Th1 cells, Th17 cell numbers in the gut are also substantially reduced in GF mice, illustrating their dependence on the gut microbiota. An astute observation about marked differences in LP Th17 cells between mice obtained from different commercial suppliers of laboratory animals garnered the attention of Dan Littman's laboratory and led to their observation that the composition of the microbiota may be an important factor in inducing LP Th17 cells. In contrast with Th1 cells, colonization of GF mice with "any old" SPF microbiota was not enough to drive the development of LP Th17 cells, suggesting that particular bacterial species were required for Th17 cell development. The laboratories of Nadine Cerf-Bensussan and Dan Littman identified a role for SFB in driving LP Th17 cell development (Gaboriau-Routhiau et al., 2009; Ivanov et al., 2009). SFB are well known to mucosal immunologists interested in the role of microbiota and immune system development and have a well-established role in intestinal IgA and T cell responses (Talham et al., 1999). Colonization of GF mice with a SFB strain led to an increase in Th17 cell numbers, which restricted the growth and invasive capacity of Citrobacter rodentium providing a mechanism by which SFB can prevent invasion by opportunistic bacteria (Ivanov et al., 2009).

Many questions still remain about how SFB drive gut Th17 cell development. Transcriptional profiling of mice colonized with SFB identified a substantial increase in the acute phase response protein serum amyloid A (SAA). SAA induced dendritic cells to produce the cytokines IL-6 and IL-23, both of which are important for Th17 development (Korn et al., 2009). How SFBs mediate this effect is unclear, however, electron micrographs suggest that SFB are in close association with the epithelium of the terminal ileum (Klaasen et al., 1992). Although some intestinal dendritic cells can extend processes into the intestinal lumen (Chieppa et al., 2006), whether SFBs interact directly with dendritic cells or with epithelial cells as go-betweens remains unclear. A striking feature of SFBs, seen on some electron micrographs, are their terminal buds (tear-drop protuberances) that occur at attachment sites with the epithelium where the SFBs appear to perturb but not penetrate host epithelial cell membranes (Klaasen et al., 1992; Ivanov et al., 2009). The whole genome sequence of the strain used in Ivanov et al. (2009) will likely prove quite informative in facilitating understanding of the host-microbiota interactions mediated by SFBs.

Bacteroides fragilis and SFBs are but a mere two out of the thousands of species populating the mouse and human intestinal tracts. The identification of these bacteria raises many questions about microbiota-immune system co-evolution and adaptation. Have only a select number of gut microbes evolved immunomodulatory potential and can a few species modulate both helper and regulatory $\mathrm{CD} 4 \mathrm{~T}$ cell behavior or have several bacterial species (or genera) evolved different effectors for different subsets? Who are the others and once identified, what are their immunomodulatory 
molecules? Are immunomodulatory species equally effective for all individuals (mouse or human) and what host genes and signaling pathways are required for these bacterial-derived signals? Some of these questions have been answered for $B$. fragilis, which also appears to modulate immunity in both mice and humans, but much remains to be discovered.

\section{LAMINA PROPRIA T REGULATORY CELLS}

An important mechanism for maintaining intestinal homeostasis is the presence of CD4 regulatory T cells (Tregs) in the intestine. Regulatory CD4 T cells are important response modulators especially in the gut which is subject to: high luminal resident bacterial loads, incursions from pathogens, changing dietary components and also Th cells which require tuning of the intensity and duration of their inflammatory responses so that chronic inflammation does not ensue.

\section{T regulatory cell subsets}

There are two main subsets of CD4 Tregs present in the gut, naturally occurring Tregs that develop in the thymus before trafficking to other sites in the body and inducible Tregs that develop in the periphery (Feuerer et al., 2009). The transcription factor Foxp3 has been defined as a master regulator of Tregs and drives both differentiation and suppressive functions. However, there is also evidence, especially in the intestine, of Tregs that do not express Foxp3. Tregs typically are able to exert their effects through dampening inflammatory responses by secreting anti-inflammatory cytokines, such as IL-10 (Maynard and Weaver, 2008). The importance of IL-10 as an inflammatory damper is evidenced by the spontaneous intestinal inflammation observed in IL-10 knock-out mice (Powrie and Leach, 1995). Interestingly, neither GF $I L-10^{-1-}$ nor SPF $I L-10^{-1-}$ mice that are not colonized with Helicobacter spp. (Kullberg et al., 1998) develop spontaneous colitis. These observations have helped to establish the important role for IL-10 in the maintenance of homeostasis between the host and its gut microbiota.

\section{Gut microbiota and T regulatory cells}

Recent studies have identified a key role for the gut microbiota in the development of intestinal Tregs. The majority of Foxp3+ intestinal Tregs are found in the LP of the SI and colon (Atarashi et al., 2011b). Studies by the laboratory of Kenya Honda have revealed that in SPF mice the frequency of Foxp3+ Tregs in the SI and colon increased after weaning, whereas peripheral Treg populations remained stable (Atarashi et al., 2011a). Comparison of SPF mice to GF mice demonstrated that while these mice have similar numbers of Tregs in the SI, GF mice have a significantly lower percentage of CD4+Foxp3+ Tregs in the colon. Furthermore, antibiotic treated mice also had significantly reduced numbers of Tregs in the colonic LP but not the SI. These findings imply that accumulation of Tregs in the colon and SI is differentially regulated and the colonic Treg differentiation is more heavily influenced by the microbiota (Atarashi et al., 2011b). Furthermore, these observations correlate with the increased number of luminal microbes in the colon vs. the SI and may suggest that inducible Tregs and the density and spatial organization of bacteria in the intestine may have coadapted.

Use of GF mice has been particularly helpful in deconstructing how single organisms and defined bacterial communities are able to instruct Treg responses. Recent work has shown that colonization of GF mice with either a single bacterial species or collection of bacterial strains can induce the differentiation of Tregs in the colon. In addition to its effects on systemic Th1 cells, B. fragilis and its immunomodulatory molecule, PSA, are capable of driving Treg differentiation (Round and Mazmanian, 2010). Monocolonization of GF mice with $B$. fragilis resulted in increased T cell expression of Foxp3+ and IL-10 in the colon, which was entirely dependent on PSA (Round and Mazmanian, 2010). Furthermore, treatment of T cells with PSA induced the development of functional Foxp3+ Tregs with suppressor activity that was likely dependent on IL-10. Importantly, PSA induced Foxp3+ Tregs were able to alleviate experimental colitis after onset of symptoms, indicating a potential use in therapeutic interventions (Mazmanian et al., 2008).

Recently Clostridium, a genus of Gram-positive bacteria that often are spore formers, have gained attention as potent inducers of gut Tregs. Investigations from the laboratory of Kenya Honda showed that while GF mice had markedly reduced colonic LP Foxp3+ Treg numbers, colonization with a collection of 46 Clostridium species restored Treg levels to that of conventionally raised mice (Atarashi et al., 2011a). There was a spatial correlation between colonic Treg distribution and the Clostridium strains along the intestine as both reach their highest density in the proximal colon (Atarashi et al., 2011a). Interestingly, the majority of Foxp3+IL-10+ Tregs induced by Clostridium were negative for Helios, which is a marker of natural (thymic-born) Tregs (Thornton et al., 2010). Therefore, the diminished Treg numbers observed in GF mice are likely inducible Tregs. Clostridium induced Tregs were functionally robust and afforded protection from intestinal inflammation in SPF mice challenged with the mucosal disruptant DSS (Atarashi et al., 2011a). Both Treg development and Treg-mediated protection from colitis were from the taxonomic Clostridium clusters IV and XIVa, which have been observed to have a reduced relative abundance in human inflammatory bowel disease patients compared with healthy controls (Frank et al., 2007). Collectively, these studies suggest that the gut microbiota may contribute to a host's resiliency from mucosa injury mediated by DSS and potentially that the absence of specific groups of bacteria may increase susceptibility to chronic inflammation. In particular, the experiments where Clostridium strains provided protection from DSS-mediated colitis raise hope that these strains hold potential as therapeutic agents.

\section{Bacteroides fragilis and Clostridium spp.: separate paths to gut Treg expansion}

Bacteroides fragilis and Clostridium spp. elicit Foxp3+IL-10+ inducible Tregs through independent mechanisms. B. fragilis, through PSA, can act directly on CD4 T cells by inducing production of TGF- $\beta 2$, which drives Treg differentiation (Round and Mazmanian, 2010). In contrast, Clostridium species influence Treg differentiation via epithelial cell-derived TGF- $\beta 1$ (Atarashi et al., 2011a). There are also differences in the signaling pathways required for Treg development between these two bacteria. Tregs isolated from TLR2 deficient mice failed to expand in response to PSA and produced low levels of IL-10, consistent with a requirement for TLR2 signaling (Round and Mazmanian, 2010). Interestingly, the effect of Clostridium was independent of several bacterial associated pattern recognition receptors, and Treg induction occurred in mice deficient in MyD88, Rip2, and Card9-mediated signaling pathways 
(Atarashi et al., 2011a). Taken together, this indicates that genera from genetically disparate phyla have evolved distinct mechanisms to influence the development of $\mathrm{T}$ cell subsets necessary for host homeostasis. These non-redundant mechanisms afford alternative opportunities for inducible Treg differentiation should genera be temporarily (or permanently) lost secondary to a course of antibiotics or an inflammatory process.

\section{CONCLUSIONS AND FUTURE DIRECTIONS}

It is intuitive that immune cells in the gut may depend on microbiota-derived cues for their development. The proximity between host and microbe in the intestine would seemingly necessitate co-adaptation. However, it has been challenging to determine the members and features of the gut microbiota that influence immune system development and function. Animal models, particularly those wherein the microbiota can be manipulated, are revealing how the gut microbiota profoundly influence the development of host intestinal immune responses. The use of GF mice, a tool that is over 50 years old (Reyniers, 1957), is providing fresh insight into the species-selectivity of gut $\mathrm{T}$ cell subset development. Much still remains to be understood about interactions of such select species (e.g., SFB and Clostridium spp.) with other gut microbial community members and the bacterial-derived signals sensed by the host. More nuanced ways to manipulate complex microbial communities will be essential to unravel host-immune interactions. Transposon-based mutagenesis strategies selectively targeting single organisms within a complex community (Goodman et al., 2009) or dynamic communities designed with

\section{REFERENCES}

Atarashi, K., Tanoue, T., Shima, T., Imaoka, A., Kuwahara, T., Momose, Y., Cheng, G., Yamasaki, S., Saito, T., Ohba, Y., Taniguchi, T., Takeda, K., Hori, S., Ivanov, Ii, Umesaki, Y., Itoh, K., and Honda, K. (2011a). Induction of colonic regulatory $\mathrm{T}$ cells by indigenous Clostridium species. Science 331, 337-341.

Atarashi, K., Umesaki, Y., and Honda, K. (2011b). Microbiotal influence on T cell subset development. Semin. Immunol. 23, 146-153.

Bandeira, A., Mota-Santos, T., Itohara, S., Degermann, S., Heusser, C., Tonegawa, S., and Coutinho, A. (1990). Localization of gamma/delta T cells to the intestinal epithelium is independent of normal microbial colonization. J. Exp. Med. 172, 239-244.

Bowman, L. M., and Holt, P. G. (2001). Selective enhancement of systemic Th1 immunity in immunologically immature rats with an orally administered bacterial extract. Infect. Immun. 69, 3719-3727.

Cerf-Bensussan, N., and GaboriauRouthiau, V. (2010). The immune system and the gut microbiota: friends or foes? Nat. Rev. Immunol. 10, 735-744.

Chieppa, M., Rescigno, M., Huang, A. Y., and Germain, R. N. (2006). Dynamic imaging of dendritic cell extension into the small bowel lumen in response to epithelial cell TLR engagement. J. Exp. Med. 203, 2841-2852. E., Mears, R., Newton, D. J., Andrew, E. M., Lawrence, B., Howell, G., Else, K. J., Gubbels, M. J., Striepen, B., Smith, J. E., White, S. J., and Carding, S. R. (2006). Intraepithelial gammadelta+ lymphocytes maintain the integrity of intestinal epithelial tight junctions in response to infection. Gastroenterology 131, 818-829.

Feng, T., Wang, L., Schoeb, T. R., Elson, C. O., and Cong, Y. (2010). Microbiota innate stimulation is a prerequisite for $\mathrm{T}$ cell spontaneous proliferation and induction of experimental colitis. $J$. Exp. Med. 207, 1321-1332.

Feuerer, M., Hill, J. A., Mathis, D., and Benoist, C. (2009). Foxp3+ regulatory T cells: differentiation, specification, subphenotypes. Nat. Immunol. 10, 689-695.

Frank, D. N., St Amand, A. L., Feldman, R. A., Boedeker, E. C., Harpaz, N., and Pace, N. R. (2007). Molecularphylogenetic characterization of microbial community imbalances in human inflammatory bowel diseases. Proc. Natl. Acad. Sci. U.S.A. 104, 13780-13785.
Dalton, J. E., Cruickshank, S. M., Egan, C.

auxotrophic mutant members (Hapfelmeier et al., 2010) offer new opportunities to deeply probe host-microbiota interactions and unravel co-adaptation in real time. Regarding the molecular crosstalk between the immune system and microbes, the discovery of the toll-like receptor and nod-like receptor signaling pathways represents a ground-breaking contribution toward understanding host-microbiota interaction. It is unlikely, however, that the dialog between the immune system and gut microbiota is limited to the current list of microbe associated molecular patterns. Gut microbes are efficient metabolic machines and their metabolites merit consideration as immune system cues. Both ATP and short chain fatty acids have emerged as microbiota-derived immune modulators - undoubtedly more bacterial immunomodulatory metabolites will be discovered (Maslowski and Mackay, 2011). Metagenomic surveys of the microbiota are providing vast amounts of information about the membership and function of the microbial world within the gut. Mucosal immunology is still discovering new $\mathrm{T}$ cell subsets and innate lymphoid cell lineages in the intestine. Thus, to paraphrase, the time is now and the place is the gut.

\section{ACKNOWLEDGMENTS}

We thank members of the Garrett laboratory, Curtis Huttenhower and members of his laboratory, and members of MiRiBA (Broad Institute) for thoughtful discussions. We thank Carey Ann Gallini for assistance with manuscript preparation, and we thank our funding sources [Burroughs Wellcome Fund, Cancer Research Institute, and the NIH (AIO78942 and CA1544260)] for their support.

Furness, J. B., Kunze, W. A., and Clerc, N. (1999). Nutrient tasting and signaling mechanisms in the gut. II. The intestine as a sensory organ: neural, endocrine, and immune responses. Am. J. Physiol. 277, G922-G928.

Gaboriau-Routhiau, V., Rakotobe, S., Lecuyer, E., Mulder, I., Lan, A., Bridonneau, C., Rochet, V., Pisi, A., De Paepe, M., Brandi, G., Eberl, G., Snel, J., Kelly, D., and CerfBensussan, N. (2009). The key role of segmented filamentous bacteria in the coordinated maturation of gut helper T cell responses. Immunity 31, 677-689.

Goodman, A. L., Mcnulty, N. P., Zhao, Y., Leip, D., Mitra, R. D., Lozupone, C. A., Knight, R., and Gordon, J. I. (2009). Identifying genetic determinants needed to establish a human gut symbiont in its habitat. Cell Host Microbe 6, 279-289.

Hapfelmeier, S., Lawson, M. A., Slack, E., Kirundi,J. K., Stoel, M., Heikenwalder, M., Cahenzli, J., Velykoredko, Y., Balmer, M. L., Endt, K., Geuking, M. B., Curtiss, R. III, McCoy, K. D., and Macpherson, A. J. (2010). Reversible microbial colonization of germ-free mice reveals the dynamics of IgA immune responses. Science 328,1705-1709.
Holt, P. G. (1995). Environmental factors and primary T-cell sensitisation to inhalant allergens in infancy: reappraisal of the role of infections and air pollution. Pediatr. Allergy Immunol. 6, 1-10.

Ismail, A. S., Behrendt, C. L., and Hooper, L. V. (2009). Reciprocal interactions between commensal bacteria and gamma delta intraepithelial lymphocytes during mucosal injury. J. Immunol. 182, 3047-3054.

Ivanov, II, Atarashi, K., Manel, N., Brodie, E. L., Shima, T., Karaoz, U., Wei, D., Goldfarb, K. C., Santee, C. A., Lynch, S. V., Tanoue, T., Imaoka, A., Itoh, K., Takeda, K., Umesaki, Y., Honda, K., and Littman, D. R. (2009). Induction of intestinal Th17 cells by segmented filamentous bacteria. Cell 139, 485-498.

Kagnoff, M. F. (1998). Current concepts in mucosal immunity. III. Ontogeny and function of gamma delta $\mathrm{T}$ cells in the intestine. Am. J. Physiol. 274, G455-G458.

Klaasen, H. L., Koopman, J. P., Poelma, F. G., and Beynen, A. C. (1992). Intestinal, segmented, filamentous bacteria. FEMS Microbiol. Rev. 8, 165-180.

Korn, T., Bettelli, E., Oukka, M., and Kuchroo, V. K. (2009). IL-17 and Th17 Cells. Annu. Rev. Immunol. 27, 485-517. 
Kullberg, M. C., Ward, J. M., Gorelick, P. L., Caspar, P., Hieny, S., Cheever, A., Jankovic, D., and Sher, A. (1998). Helicobacter hepaticus triggers colitis in specific-pathogen-free interleukin-10 (IL-10)-deficient mice through an IL-12- and gamma interferondependent mechanism. Infect. Immun. 66, 5157-5166.

Lee, Y. K., Mukasa, R., Hatton, R. D., and Weaver, C. T. (2009). Developmental plasticity of Th17 and Treg cells. Curr. Opin. Immunol. 21, 274-280.

Maslowski, K. M., and Mackay, C.R. (2011). Diet, gut microbiota and immune responses. Nat. Immunol. 12, 5-9.

Maynard, C. L., and Weaver, C. T. (2008). Diversity in the contribution of interleukin-10 to T-cell-mediated immune regulation. Immunol. Rev. 226, 219-233.

Mazmanian, S. K., Liu, C. H., Tzianabos, A. O., and Kasper, D. L. (2005). An immunomodulatory molecule of symbiotic bacteria directs maturation of the host immune system. Cell 122, 107-118.

Mazmanian, S. K., Round, J. L., and Kasper, D. L. (2008). A microbial symbiosis factor prevents intestinal inflammatory disease. Nature 453, 620-625.

Mosmann, T. R., Cherwinski, H., Bond, M. W., Giedlin, M.A., and Coffman, R. L. (1986). Two types of murine helper $\mathrm{T}$ cell clone. I. Definition according to profiles of lymphokine activities and secreted proteins. J. Immunol. 136, 2348-2357.

Powrie, F., and Leach, M. W. (1995). Genetic and spontaneous models of inflammatory bowel disease in rodents: evidence for abnormalities in mucosal immune regulation. Ther. Immunol. 2, 115-123.

Reyniers, J. A. (1957). The production and use of germ-free animals in experimental biology and medicine. Am. J. Vet. Res. 18, 678-687.

Roberts, S. J., Smith, A. L., West, A. B., Wen, L., Findly, R. C., Owen, M. J., and Hayday, A.C. (1996). T-cell alpha beta+ and gamma delta+ deficient mice display abnormal but distinct phenotypes toward a natural, widespread infection of the intestinal epithelium. Proc. Natl. Acad. Sci. U.S.A. 93, 11774-11779.

Round, J.L., and Mazmanian, S. K. (2009). The gut microbiota shapes intestinal immune responses during health and disease. Nat. Rev. Immunol. 9,313-323.

Round, J. L., and Mazmanian, S. K. (2010). Inducible Foxp3+ regulatory T-cell development by a commensal bacterium of the intestinal microbiota. Proc. Natl. Acad. Sci. U.S.A. 107, 12204-12209.

Sears, C. L., and Pardoll, D. M. (2011). Perspective: alpha-bugs, their microbial partners, and the link to colon cancer. J. Infect. Dis. 203, 306-311.

Sellge, G., Magalhaes, J. G., Konradt, C., Fritz, J. H., Salgado-Pabon, W.,
Eberl, G., Bandeira, A., Di Santo, J. P., Sansonetti, P. J., and Phalipon, A. (2010). Th17 cells are the dominant T cell subtype primed by Shigella flexneri mediating protective immunity. $J$. Immunol. 184, 2076-2085.

Sheridan, B. S., and Lefrancois, L. (2010). Intraepithelial lymphocytes: to serve and protect. Curr. Gastroenterol. Rep. $12,513-521$.

Smith, K., Mccoy, K. D., and Macpherson, A. J. (2007). Use of axenic animals in studying the adaptation of mammals to their commensal intestinal microbiota. Semin. Immunol. 19, 59-69.

Talham, G. L., Jiang, H. Q., Bos, N. A., and Cebra, J. J. (1999). Segmented filamentous bacteria are potent stimuli of a physiologically normal state of the murine gut mucosal immune system. Infect. Immun. 67, 1992-2000.

Thornton, A. M., Korty, P. E., Tran, D. Q., Wohlfert, E. A., Murray, P. E., Belkaid, Y., and Shevach, E. M. (2010). Expression of Helios, an Ikaros transcription factor family member, differentiates thymic-derived from peripherally induced Foxp $3+$ T regulatory cells. J. Immunol. 184, 3433-3441.

van Wijk, F., and Cheroutre, H. (2009). Intestinal T cells: facing the mucosal immune dilemma with synergy and diversity.Semin. Immunol. 21, 130-138.

Wilson, C. B. (1986). Immunologic basis for increased susceptibility of the neonate to infection. J. Pediatr. 108, 1-12.
Yang, H., Antony, P. A., Wildhaber, B. E., and Teitelbaum, D. H. (2004) Intestinal intraepithelial lymphocyte gamma delta-T cell-derived keratinocyte growth factor modulates epithelial growth in the mouse. J. Immunol. 172, 4151-4158.

Zhu, J., Yamane, H., and Paul, W.E. (2010). Differentiation of effector CD4 T cell populations $\left({ }^{*}\right)$. Annu. Rev. Immunol. $28,445-489$.

Conflict of Interest Statement: The authors declare that the research was conducted in the absence of any commercial or financial relationships that could be construed as a potential conflict of interest.

Received: 01 April 2011; paper pending published: 14 April 2011; accepted: 07 May 2011; published online: 26 May 2011. Citation: Smith PM and Garrett WS (2011) The gut microbiota and mucosal T cells. Front. Microbio. 2:111. doi: 10.3389/ fmicb.2011.00111

This article was submitted to Frontiers in Cellular and Infection Microbiology, a specialty of Frontiers in Microbiology. Copyright $\odot 2011$ Smith and Garrett. This is an open-access article subject to a nonexclusive license between the authors and Frontiers Media SA, which permits use, distribution and reproduction in other forums, provided the original authors and source are credited and other Frontiers conditions are complied with. 